\title{
Método para el aseguramiento de ingresos basado en análisis de riesgos y computación con palabras
}

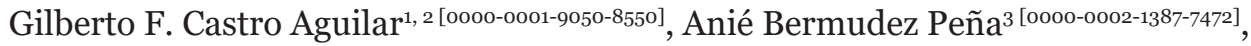 \\ Francisco G. Palacios Ortiz², Fausto R. Orozco Lara², Diana J. Espinoza Villón², \\ Diana M. López Álvarez [oooo-ooo3-2457-7683]
}

gilberto.castro@cu.ucsg.edu.ec, gilberto.castroa@ug.edu.ec, abp@uci.cu, francisco. palacioso@ug.edu.ec, fausto.orozcol@ug.edu.ec, diana.espinozavi@ug.edu.ec, dlopez@ ecotec.edu.ec

${ }^{1}$ Facultad de Ingeniería, Universidad Católica Santiago de Guayaquil, Ecuador.

${ }^{2}$ Facultad de Ciencias Matemáticas y Físicas, Universidad de Guayaquil, Ecuador.

${ }^{3}$ Departamento de Gestión de Proyectos, Universidad de las Ciencias Informáticas, La Habana, Cuba.

${ }^{4}$ Facultad de Ingeniería en Sistemas Computacionales y Telecomunicaciones, Universidad ECOTEC, Guayaquil, Ecuador.

DOI: 10.17013/risti.27.126-140

Resumen: El incremento de la competitividad en los mercados globales ha provocado la necesidad de perfeccionamientos en las organizaciones orientadas a proyectos, dirigidas a mejorar su salud financiera. El aseguramiento de ingresos combina técnicas de estadística, gestión de riesgos, alcance, tiempo, soft computing y minería de datos anómalos, orientado a la reducción de costos y la maximización de ingresos en las organizaciones. Este trabajo presenta un método para el aseguramiento de ingresos basado en técnicas de análisis de riesgos y computación con palabras. Con ello se persigue mejorar los procesos de detección y prevención de situaciones que afectan los ingresos en las organizaciones orientadas a proyectos. Se desarrolla la gestión de riesgos con un enfoque proactivo para la planificación y la evaluación cualitativa de riesgos. Se realiza un análisis final que muestra las ventajas de la propuesta con respecto a los resultados obtenidos con la técnica tradicional del PMBOK.

Palabras-clave: Análisis de riesgos; aseguramiento de ingresos; computación con palabras; organizaciones orientadas a proyectos; toma de decisiones.

\section{Revenue Assurance Method using Risk Analysis and Computing with Words}

Abstract: The increase in competitiveness in global markets has led to the need for improvements in project-oriented organizations aimed at stimulating financial health. Revenue assurance combines statistical techniques, risk management, scope, time, soft computing, and anomalous data mining, with goals of reducing costs and maximizing revenue in the organizations. This paper presents a method 


\begin{abstract}
for revenue assurance based on risk analysis techniques and word computation. Furthermore, this aims at improving the detection and prevention processes of situations that affect revenue in project-oriented organizations. Risk management is developed with a proactive approach for planning and qualitative risk assessment. A final analysis is carries out that shows the great advantages of the proposal with respect to the results obtained with the traditional PMBOK technique.
\end{abstract}

Keywords: Computing with words; decision-making; project management organizations; revenue assurance; risk analysis.

\title{
1. Introducción
}

Los problemas de dirección han estado siempre presentes en el desarrollo de la sociedad. Una de las formas de organización que ha ganado fuerza por su aplicabilidad en diferentes escenarios, es la Dirección Integrada de Proyectos, que ha dado lugar a que crezca la cantidad de organizaciones orientadas a proyectos (Delgado, 2011). Dichas organizaciones son aquellas que desarrollan nuevos productos o servicios, estableciendo sus recursos en forma de proyectos con fechas de inicio y fin bien determinadas (PMI, 2017).

En este tipo de organizaciones, a pesar de los esfuerzos por mejorar la eficiencia y la eficacia en la gestión, persisten numerosas dificultades y situaciones que generan pérdidas de ingresos. Un estudio realizado por The Standish Group International Incorporated, arrojó como resultado que históricamente las cifras de proyectos satisfactorios, renegociados y cancelados, se ha movido por alrededor del $29 \%, 52 \%$ y $19 \%$ respectivamente (SGI, 2014; SGI, 2015). Un elemento importante que debe ser analizado más allá de la cantidad de proyectos renegociados o fracasados, es el impacto económico y social de los mismos.

En las organizaciones orientadas a proyectos se planifican múltiples proyectos simultáneamente. Durante el desarrollo de los planes se introducen defectos que pueden afectar significativamente los costos previstos para la ejecución del proyecto, provocados por errores humanos o enmascarando procesos de corrupción y fraude. Existe un conjunto de errores en la gestión de proyectos que tiene un alto impacto en el éxito de los mismos y por ende en el aseguramiento de ingresos en las organizaciones orientadas a proyectos (Phillips, 2013; Leach, 2014; Schwalbe, 2015; Verzuh, 2015). Entre los problemas más comunes se señalan:

- Errores en la definición del alcance del proyecto, que generalmente provoca malas estimaciones en los costos, afectando los ingresos y las utilidades.

- Errores en la planificación, control y seguimiento de los proyectos, respecto al cubrimiento parcial de los requisitos definidos en el alcance o debido a que el plan no es comprendido por los miembros de la organización.

- Poca atención a los riesgos del proyecto, que con frecuencia provoca un sobregiro en los costos del proyecto, afectando los ingresos y las utilidades.

- Deficiente gestión de cambios que provoca aumentos o modificaciones significativas en el alcance del proyecto, afectando los ingresos y las utilidades.

- Empleo de la tecnología equivocada, en el caso de los proyectos de tecnologías de la información, se refleja en malas decisiones arquitectónicas que generan atrasos, generan el re-trabajo y afectan las utilidades de las organizaciones. 
Como estrategia para ayudar a resolver algunos de estos problemas, el presente trabajo propone apoyar el aseguramiento de ingresos aplicando análisis de riesgos y computación con palabras en organizaciones orientadas a proyectos.

\section{Marco teórico}

\subsection{Aseguramiento de ingresos}

El aseguramiento de ingresos como área de conocimiento surge desde finales de la década de 1970, en el sector de las telecomunicaciones, como disciplina orientada a la protección y recuperación de los recursos financieros de las organizaciones. En este contexto se han establecido definiciones para describir el objeto de estudio de esta disciplina. Entre ellas se destaca Khan quien plantea que "el aseguramiento de ingresos es el conjunto de actividades que son aplicadas para asegurar que los procesos del negocio, la estructura organizacional, los controles y los sistemas de información, relacionadas con el ciclo de ingresos de las organizaciones, trabajen juntos con efectividad" (Khan, 2014).

Con el desarrollo de la disciplina de aseguramiento de ingresos surgen y se fortalecen espacios para la formación de especialistas y la creación de estándares: TM Forum (TMForum, 2015) y la Asociación Global de Profesionales de Aseguramiento de Ingresos GRAPA (Mattison, 2005).

GRAPA es fundada con el objetivo de definir, estandarizar y profesionalizar el trabajo de aseguramiento de ingresos; además de ofrecer ayuda y proveer soporte en todo el mundo a los profesionales de esta área de conocimiento (GRAPA, 2017). Plantea que entre las principales causas que provocan pérdida de ingresos en las telecomunicaciones se encuentran la formación del personal, la integración de los procesos en la organización y la adopción de estándares. Esta situación es similar en las organizaciones orientadas a proyectos, pero con la diferencia que los costos, presupuestos y planificación ganan en relevancia. Según GRAPA, entre las técnicas más empleadas en el aseguramiento de ingresos se encuentran los análisis de: riesgos, intercambio, procesos, sistemas y estadístico.

TM Forum promueve un modelo de evaluación de la madurez de las organizaciones en la implantación de los procesos de aseguramiento de ingresos. Dicho modelo de madurez tiene cinco niveles: inicial, repetible, definido, manejado y optimizado. Plantea que entre los principales factores que influyen en la madurez de los procesos de aseguramiento de ingresos, se encuentran el nivel de experiencia de los equipos de aseguramiento y las tecnologías, ambos con un 23 \% de relevancia. TM Forum ubica en el mismo nivel las actividades de recuperación de ingresos y las acciones para el monitoreo y el control de los riesgos del aseguramiento (TMForum, 2015).

Uno de los planteamientos tradicionales para definir una buena gestión de la organización es conseguir alinear las tecnologías de la información (TI) con el negocio. Para ello es necesario definir una robusta y clara estructura de gobierno corporativo basado en las TI como parte indivisible. En (Prieto, 2015) se propone un marco de mejora continua de gobierno TI para las entidades financieras.

Después de un estudio realizado, se verificó que las técnicas para el aseguramiento de ingresos a nivel mundial aún son insuficientes tanto para las empresas de 
telecomunicaciones donde surgen, como para las organizaciones orientadas a proyectos. Entre las principales deficiencias de esta disciplina se señalan (Mattison, 2005; Acosta, 2008; Khan, 2014; TMForum, 2015; GRAPA, 2017):

- Dependen de recursos humanos para su aplicación, que éstos a su vez, también están sujetos a posibles errores de operación y no se maneja la certidumbre de las decisiones, ni la detección de situaciones anómalas.

- En las organizaciones orientadas a proyectos, se presentan fenómenos como la heterogeneidad en los datos, la imprecisión y la incertidumbre; que las técnicas tradicionales de aseguramiento de ingresos no gestionan adecuadamente.

- Con frecuencia las soluciones planteadas se basan solamente en enfoques reactivos y no usan adecuadamente estrategias activas o proactivas del aseguramiento de ingresos.

- Muchas de las soluciones para el aseguramiento de ingresos se basan en los sistemas de reglas de producción. Este tipo de sistema basado en el conocimiento presenta dificultades para tratar con el dinamismo y la diversidad de las organizaciones orientadas a proyectos, cubrimiento del dominio y visión parcial del espacio de búsqueda, lento reaprendizaje y poco nivel de reutilización.

Con frecuencia las soluciones implantadas constituyen cajas negras soportadas por herramientas privativas que afectan la soberanía tecnológica de las organizaciones. No se sabe a ciencia cierta todo el impacto que tiene para la organización la gestión de la información con estas herramientas externas.

Se ha identificado que muchos de estos problemas afectan la eficiencia y la eficacia de los procesos de aseguramiento de ingresos desde la perspectiva de la capacidad de detección de situaciones anómalas.

\subsection{Influencia de las escuelas de gestión de proyectos en el aseguramiento de ingresos}

La disciplina de Gestión de Proyectos está instituida por escuelas o instituciones dedicadas a la formalización de métodos de organización y trabajo. Entre estas instituciones se encuentran el Project Management Institute (PMI) con su estándar PMBOK (PMI, 2017), el Software Engineering Institute (SEI) con el estándar Capability Maturity Model Integration (CMMI) (SEI, 2010), el International Project Management Association (IPMA, 2016) y la ISO con sus normas 10006 y 21500 (Zandhuis, 2012).

Tradicionalmente las escuelas de gestión de proyectos como PMBOK y CMMI han tenido una fuerte orientación predictiva. Es decir, a partir del detalle del producto que se quiere elaborar, se planifican actividades en base a los recursos disponibles (Vondran, 2015). Con dicha proyección inicial, el objetivo durante el proyecto es conseguir que se cumpla lo previsto: calendario, costes y calidad. En cambio, los proyectos gestionados con metodologías ágiles se inician sin un detalle cerrado de lo que va a ser construido. En (Mathkour, 2008) se presenta una aplicación de gestión de riesgos en el desarrollo de proyectos de software que usan la metodología ágil Extreme Programming (XP). En (Schnoeller, 2016) se presenta una estrategia basada en la adquisición de conocimiento para la gestion de riesgos aplicando XP en el desarrollo de software distribuido. 
La Tabla 1 ofrece un análisis tanto del PMBOK como de la ISO 21500 respecto a los procesos que influyen con mayor fuerza en el aseguramiento de ingresos desde el punto de vista de la gestión de los riesgos. En la misma, se relacionan las técnicas que se proponen en estos procesos. Se puede apreciar que, para ambas normas, existe convergencia en las técnicas y buenas prácticas para la gestión de riesgos.

Aunque estas normas incluyen actividades y técnicas que pueden influir en el aseguramiento de ingresos, están mayormente basadas en análisis manuales con una fuerte influencia de expertos. El mayor aporte de estas metodologías al aseguramiento de ingresos se puede encontrar en el área de gestión de riesgos que constituyen técnicas de análisis proactivo. No obstante, se señala que las propias técnicas que proponen para el análisis cualitativo son rígidas y no permiten un adecuado tratamiento de la incertidumbre y la ambigüedad existente en los procesos de gestión de riesgos.

\begin{tabular}{|c|c|c|}
\hline PMBOK & ISO 21500 & $\begin{array}{l}\text { Técnicas que proponen que pueden influir en el } \\
\text { aseguramiento de ingresos }\end{array}$ \\
\hline 11.2 Identificar riesgos & $\begin{array}{l}4.3 .28 \text { Identificar } \\
\text { riesgos }\end{array}$ & $\begin{array}{l}\text {-Juicio de expertos, análisis FODA, lista de verificación. } \\
\text {-Técnicas de diagramación: diagramas de causa y efecto, } \\
\text { diagramas de flujo de procesos, diagramas de influencias. } \\
\text {-Técnicas de recopilación de información. }\end{array}$ \\
\hline $\begin{array}{l}\text { 11.3 Realizar el análisis } \\
\text { cualitativo } \\
\text { 11.4 Realizar el análisis } \\
\text { cuantitativo }\end{array}$ & $\begin{array}{l}4.3 .29 \text { Evaluar } \\
\text { riesgos }\end{array}$ & $\begin{array}{l}\text {-Juicio de expertos. } \\
\text {-Evaluación de probabilidad e impacto de los riesgos. } \\
\text {-Evaluación de la calidad de los datos sobre riesgos. } \\
\text {-Categorización de riesgos y priorización. } \\
\text {-Técnicas de análisis cuantitativo: análisis de sensibilidad, } \\
\text { árboles de decisión, modelado y simulación. }\end{array}$ \\
\hline $\begin{array}{l}11.5 \text { Planear la respuesta } \\
\text { a los riesgos }\end{array}$ & $\begin{array}{l}4 \cdot 3 \cdot 30 \text { Tratar los } \\
\text { riesgos }\end{array}$ & $\begin{array}{l}\text {-Juicio de expertos. } \\
\text {-Estrategias para riesgos negativos o amenazas. } \\
\text {-Estrategias para riesgos positivos u oportunidades. } \\
\text {-Estrategias de respuesta a contingencias. }\end{array}$ \\
\hline 11.6 Controlar riesgos & $\begin{array}{l}4 \cdot 3 \cdot 31 \text { Controlar } \\
\text { los riesgos }\end{array}$ & $\begin{array}{l}\text {-Revaluación y auditorías a los riesgos. } \\
\text {-Análisis de variación, tendencias y análisis de reserva. } \\
\text {-Medición del desempeño técnico. }\end{array}$ \\
\hline
\end{tabular}

Tabla 1 - Procesos de PMBOK e ISO 21500 asociados a la gestión de riesgos en el aseguramiento de ingresos

El SEI en CMMI (SEI, 2010) presenta un estándar diseñado para medir la capacidad y madurez de organizaciones orientadas a proyectos de software, pero al igual que PMBOK en su definición no incorpora suficientes elementos asociados a técnicas de minería de datos u optimización que puedan contribuir de esa forma en la detección de situaciones anómalas generadoras de pérdidas de ingresos en las organizaciones.

CMMI relaciona las prácticas genéricas y específicas aplicables a la gestión de riesgos. Pero, no propone algoritmos concretos para lograrlo. Tampoco propone el uso de técnicas de minería de datos, que ayuden a detectar situaciones anómalas, en la gestión de la organización. Se centra en el trabajo manual y la documentación exhaustiva de 
los procesos, más que en la determinación de fallas y errores a partir del análisis de los datos.

Como estrategia para resolver estos problemas en organizaciones orientadas a proyectos, los autores, desarrollan investigaciones asociadas a la aplicación de técnicas de minería de datos (Castro, 2017).

\section{Método para el aseguramiento de ingresos basado en análisis de riesgos y computación con palabras}

El método que se propone para el aseguramiento de ingresos en organizaciones orientadas a proyectos, consta de tres partes fundamentales: comprensión de la organización, gestión proactiva de riesgos y evaluación para la toma de decisiones.

\subsection{Comprensión de la organización}

Inicialmente en la investigación se realiza una comprensión de la organización y diagnóstico de sus proyectos. Esta primera etapa se centra en comprender los procesos de la organización para definir una taxonomía que ayude a identificar las situaciones anómalas que afecten los ingresos, como posibles causas de fallos, malas planificaciones, fraudes, errores en la ejecución de los proyectos o simplemente fugas de ingresos.

Un conjunto de expertos construye la secuencia de actividades primarias y de soporte que forman la cadena de valor de la organización. A partir del análisis de la cadena de valor, se construye una matriz DAFO identificando elementos que influyen en cada actividad primaria tanto en la reducción de los costos como en la mejora de los ingresos y que afectan el margen de cada actividad.

En esta identificación y evaluación de elementos que influyen en los ingresos de la organización, se identifican seis grupos:

1. Nuevos servicios o productos, generadores de nuevos ingresos.

2. Fuentes de errores que afectan el aprovechamiento de las oportunidades o que generan pérdidas de ingresos.

3. Errores o posibles situaciones anómalas que potencien las amenazas afectando los ingresos.

4. Actividades para mitigar o evitar las amenazas.

5. Riesgos externos y fuentes externas que pueden provocar pérdidas de ingresos.

6. Actividades basadas en las fortalezas que ayuden a evitar o mitigar las pérdidas de ingresos por amenazas.

Estos seis grupos permiten a los expertos evaluar cada elemento que influye en los ingresos, agrupados por su naturaleza. Para mitigar, evitar o potenciar cada elemento en función de su impacto en los ingresos, los expertos proponen un conjunto de acciones a ejecutar. Generalmente acciones de grupos diferentes pueden ejecutarse en paralelo. Los elementos de los grupos 2, 3 y 5 concentran los factores internos o externos, las posibles fuentes de errores y otras situaciones anómalas que afectan los ingresos. Los grupos 4 y 6 constituyen acciones recomendadas y que serán empleadas siguiendo un enfoque proactivo en la etapa 2 del método propuesto. El grupo 1 están orientados a 
explotar las oportunidades y serán considerados siguiendo un enfoque proactivo en la etapa 2 de este método.

\subsection{Gestión de riesgos con enfoque proactivo}

En esta segunda etapa del método propuesto, se aplican las técnicas y procesos del PMBOK. Además, se introduce una técnica nueva en los procesos de análisis cualitativo de los riesgos. En particular se propone una técnica basada en el modelo de computación con palabras 2-tuplas (Herrera, 2000; Duran , 2017) en lugar de la técnica propuesta por el PMBOK.

Algoritmo utilizado para la evaluación de los riesgos usando el modelo de 2-tuplas:

1. Seleccionar el conjunto de $m$ expertos. Este conjunto estará denotado por $E$, identificándose el i-ésimo como $e_{i}$.

2. Identificar los riesgos con impacto en el aseguramiento de ingresos aplicando las técnicas del PMBOK.

3. Establecer los criterios de evaluación de riesgos. En este caso se toman: probabilidad de ocurrencia, impacto y facilidad de detección.

4. Evaluar cada uno de los riesgos identificados empleando técnicas de computación con palabras. Se define un conjunto básico de términos lingüísticos (LBTL) para la evaluación de los elementos, basado en el grado de impacto en los ingresos ya sea positiva o negativamente. LBTL $=\{$ Ninguno, Muy bajo, Bajo, Medio, Alto, Muy alto, Perfecto\}, ver Figura 1. Los expertos evalúan cada elemento según la Tabla 2.

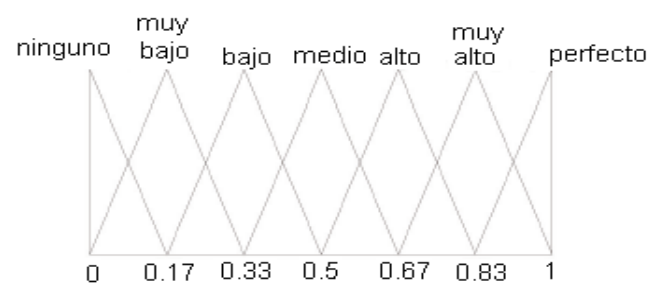

Figura 1 - Variable lingüística "Evaluación del impacto del riesgo"

\begin{tabular}{|c|c|c|c|c|c|c|c|}
\hline \multirow[t]{3}{*}{ Riesgos } & \multicolumn{7}{|c|}{ Expertos } \\
\hline & $e_{1}$ & & & $\cdots$ & $e_{m}$ & & \\
\hline & $\mathrm{P}$ & I & $\mathrm{D}$ & & $\mathrm{P}$ & $\mathrm{D}$ & I \\
\hline Riesgo 1 & $\mathrm{X} 11 \mathrm{P}$ & $\mathrm{X} 11 \mathrm{I}$ & $\mathrm{X} 11 \mathrm{D}$ & $\cdots$ & $\mathrm{X} 1 \mathrm{mP}$ & $\mathrm{X} 1 \mathrm{mI}$ & $\mathrm{X} 1 \mathrm{mD}$ \\
\hline$\vdots$ & $\vdots$ & $\vdots$ & $\vdots$ & $\vdots$ & & & $\vdots$ \\
\hline Riesgo n & $\mathrm{Xn} 1 \mathrm{P}$ & Xn1I & $\mathrm{Xn} 1 \mathrm{D}$ & $\ldots$ & $\mathrm{XnmP}$ & XnmI & $\mathrm{XnmD}$ \\
\hline
\end{tabular}

Tabla 2 - Evaluación de riesgos aplicando computación con palabras

Donde P es la probabilidad, I el impacto y D la facilidad de detección del riesgo. 
5. Siguiendo el modelo 2-tuplas de computación con palabras se agregan las evaluaciones de los expertos consolidando las mismas por cada elemento a evaluar.

6. Al finalizar se tienen los riesgos identificados y su impacto en los procesos de aseguramiento de ingresos.

\subsection{Evaluación de resultados para la toma de decisiones}

En el método propuesto, finalmente se evalúan los resultados, se estima el impacto para la organización de los riesgos detectados y se realiza un análisis detallado. Al llegar a este paso se cuenta con dos grupos de situaciones dirigidas a la recuperación de ingresos:

- Conjunto de riesgos que son evitados o mitigados, permitiendo disminuir la fuga de ingresos.

- Conjunto de medidas proactivas para mitigar o eliminar los riesgos. Estas tienen un costo de implementación que debe considerarse en el aseguramiento de ingresos.

Para estimar el impacto económico de estas situaciones, se propone a continuación un conjunto de técnicas.

Técnica de estimación por tres valores: Se basa en lograr estimar tres valores: monto más probable (M) a ser recuperado basado en una evaluación realista del experto, monto a recuperar basado en un enfoque optimista $(\mathrm{O})$ tomando como base el mejor escenario posible y el monto a recuperar pesimista $(\mathrm{P})$ basado en el análisis del peor escenario para la recuperación de los ingresos. Luego se procede a calcular el valor estimado usando la Ec. (1).

$$
c e=\frac{O+4 M+P}{6}
$$

Técnica de estimación ascendente: En este caso se realiza una estimación de lo recuperado por cada una de los riesgos detectados y se procede a consolidar este resultado sumando los montos en unidades monetarias asociados a cada actividad. En caso de que sea difícil la estimación del impacto de una actividad se procede a descomponer la misma en componentes de nivel inferior para un análisis más detallado y luego se consolidan las estimaciones respetando la jerarquía construida.

Técnica basada en el análisis de redes: Esta técnica se basa en la construcción de una red de forma tal que cada nodo representa un estado posible de la organización ante diferentes situaciones de riesgos. Las aristas representan las posibles decisiones y cada arista está etiquetada por un vector con las siguientes características (Johnsonbaugh, 1999; Merigó, 2013).

- La primera componente representa la probabilidad de tomar la decisión o de que ocurra un riesgo.

- La segunda componente representa alguno de los siguientes elementos: costo de tomar la decisión, impacto económico positivo en caso de ocurrir una oportunidad, impacto económico negativo en caso de ocurrir una amenaza.

Con esta estructura se puede aplicar un conjunto de técnicas clásicas asociadas al trabajo con redes que permiten estimar el impacto económico de diferentes escenarios del 
aseguramiento de ingresos, entre las que se encuentran: redes bayesianas, algoritmo de flujo máximo, algoritmo de Dijkstra, algoritmo de Floyd para determinar el camino con costo mínimo de la organización.

Para realizar la toma de decisiones se propone el uso de sistemas de información combinado con el juicio de expertos. Se deben seguir las siguientes recomendaciones:

- Aplicar sistemas de información que permitan la gestión por cortes y el uso de indicadores objetivos que cubran las áreas de conocimiento.

- Involucrar a los miembros en la búsqueda de las soluciones.

- Priorizar en la toma de decisiones las actividades de la cadena de valor con mayor impacto en los ingresos y las utilidades.

En este paso también pueden ser empleados sistemas de recomendaciones entre otras técnicas de computación emergente.

\section{Evaluación y resultados}

Para aplicar la propuesta, esta se implementó sobre el sistema de información de la plataforma GESPRO por la versatilidad de la misma y la gran cantidad de funcionalidades para el aseguramiento de ingresos que incluye (Castro, 2016), entre las que se encuentran:

- Módulo para el análisis de datos y el aseguramiento de ingresos, que integra bibliotecas en $\mathrm{R}$ para la detección de datos anómalos.

- Módulo para la gestión de riesgos, aplicable para el análisis proactivo.

- Cuadro de mando con indicadores y alertas tempranas, orientado a la detección de insuficiencias en la planificación y la ejecución de proyectos.

- Gestión del alcance y de la calidad respecto al cubrimiento de los requisitos en el cronograma y el control de la calidad.

- Gestión de los costos de los proyectos y predicción de costos de proyectos en función del comportamiento de los datos.

La Figura 2 muestra el módulo de gestión de riesgos como parte del subsistema de aseguramiento de ingresos en la herramienta GESPRO. Nótese que la herramienta

\begin{tabular}{|c|c|c|c|c|c|c|c|c|c|}
\hline \multicolumn{2}{|r|}{ CATEGORIAS DE RIESGOS } & \multicolumn{2}{|c|}{ ESTADOS DE RIESGOS } & RIESGOS & & & & & \\
\hline \multicolumn{4}{|c|}{ Riesgos del Centro } & & & & & (4) Nuevo riesgo & (9) Ayuda \\
\hline \multicolumn{10}{|c|}{$\rightarrow$ Filtros } \\
\hline$\#$ & Nombre & & Prob. & Impacto & Detec. & Exposición & Evaluaciones & & \\
\hline 2 & Pérdila de recursos & humanos & $(B a j o ; 0.0)$ & (Alto; 0.0) & (Muy alto; 0.0 ) & (Medlo; 0.0) & (4) 1 Ver & 2 Modificar & 耍 Borrar \\
\hline 1 & Planificación sobre & e costos & (Medlo; 0) & (Medlo; 0) & (Medlo; 0) & (Medlo; 0.0 ) & (4) 0 & 2 Modificar & 面 Borrar \\
\hline 3 & Pérdlda de Recursos & $s$ Humanos & (Alto; 0.0 ) & (Alto; 0.0 ) & (Medio; 0.0) & (Medio; 0.0) & (4) 2 Ver & 2 Modificar & 面 Borrar \\
\hline 4 & Dificultades con fenc & ómenos ... & (Bajo; 0.0) & (Alto; 0.0) & $(\mathrm{Bajo} ; 0.0)$ & (Medlo; 0.0 ) & (4) 1 \&er & Modificar & 耍 Borrar \\
\hline
\end{tabular}

Figura 2 - Vista de gestión de riesgos en la plataforma GESPRO como parte del subsistema de aseguramiento de ingresos 
permite evaluar los riesgos según varios criterios y ser realizado por múltiples expertos. Además, se permite exportar a fichero CSV la información correspondiente al estado actual de la evaluación de los riesgos del proyecto.

En la validación del método propuesto se aplican técnicas de triangulación metodológica de métodos y de expertos. Se compara el enfoque proactivo propuesto para la gestión de riesgos contra la técnica del PMBOK. Para ellos, se realiza un experimento que compara la técnica tradicional propuesta en el PMBOK con la técnica basada en el modelo 2-tuplas de computación con palabras.

Para la experimentación se empleó una base de datos de proyectos terminados del repositorio perteneciente al Laboratorio de Investigaciones en Gestión de Proyectos (Castro, 2016; Castro, 2017). Dicha base de datos cuenta con 14 proyectos reales de desarrollado de software, los cuales están concluidos y se conoce cómo se comportaron sus riesgos, o sea se tiene un patrón de comparación con lo que realmente ocurrió con los proyectos. La información de lo que realmente ocurrió con los proyectos se considera como la respuesta deseada que deben aportar los métodos de evaluación cualitativa que se comparan en el trabajo.

Para la validación se tomó un grupo de 6 expertos en gestión de proyectos que no participaron en los proyectos seleccionados. A continuación se caracterizan los expertos consultados para la evaluación de los riesgos: 4 Doctores y 2 Máster, respecto a los años dedicados: 18.8 como promedio, 9.8 desviación estándar, 14 años mínimos y 37 años máximos dedicados. A los expertos se les suministró suficiente información que caracteriza a cada proyecto para que realizaran la evaluación de los riesgos. Luego se procedió a aplicar los siguientes pasos:

- Paso 1. Los expertos sentados en una mesa de trabajo, evaluaron por consenso cada riesgo en cada proyecto empleando el método propuesto por el PMBOK al que llamaremos Riesgos-PMBOK.

- Paso 2. Los expertos de forma independiente, evaluaron según su criterio cada riesgo en cada proyecto empleando el método propuesto en este trabajo. Aplicaron técnicas de computación con palabras, empleando la variable representada en la Figura 1, a este método lo llamamos al Riesgos-CWW.

- Paso 3. Se calculó el error cuadrático medio cometido en el proyecto p (Ep) según las dos variantes de métodos de evaluación, ver Ec. (2).

$$
E_{p}=\frac{1}{18} \sum_{i=1}^{18}\left(D_{i}-Y_{i}\right)^{2}
$$

Donde $\mathrm{D}_{\mathrm{i}}$ es lo que realmente ocurrió en los riesgos, o sea la salida deseada.

Siendo $\mathrm{Y}_{\mathrm{i}}$ la salida dada por los diferentes métodos de evaluación ante cada riesgo.

Se tuvieron en cuenta 18 de los riesgos más comunes en este escenario, que cubren todas las áreas de conocimiento de la gestión de proyectos, ver Tabla 3.

Finalmente se compararon los errores cuadráticos medios calculados según cada método para cada uno de los proyectos, ver resultados en la Tabla 4 y Figura 3. 


\begin{tabular}{|c|c|}
\hline Riesgos & Área de conocimiento \\
\hline Dificultades energéticas, afectan la producción & \multirow{3}{*}{ Adquisiciones } \\
\hline Dificultades con el transporte, afectan el plan & \\
\hline Aumento de los precios de los recursos & \\
\hline Dificultades con la licitación de los requisitos & \multirow{2}{*}{ Alcance y Calidad } \\
\hline Dificultades con la definición de la arquitectura & \\
\hline Falta de liderazgo en los jefes del proyecto & Integración \\
\hline Trámites engorrosos para la comercialización & Interesados \\
\hline Atrasos en entrega de los proveedores & Interesados y Adquisiciones \\
\hline Tardía entrega de información por cliente & Interesados y Alcance \\
\hline Cliente desinteresado que no participa en los encuentros & Interesados y Comunicaciones \\
\hline Pérdida de recursos humanos, condiciones & \multirow{4}{*}{ Recursos humanos } \\
\hline Bajo nivel de formación de recursos humanos & \\
\hline Pocos incentivos al equipo, baja producción & \\
\hline Mala conformación de equipos & \\
\hline Fenómenos atmosféricos, afectan la productividad & \multirow{2}{*}{ Riesgos } \\
\hline Rotura de equipos y lento mantenimiento & \\
\hline Bajos niveles de reutilización & Tiempo y Calidad \\
\hline Elevada presión externa, provoca errores de planificación y ejecución & Tiempo, Integración, Calidad \\
\hline
\end{tabular}

Tabla 3 - Relación de áreas de conocimientos y riesgos identificados para la validación

\begin{tabular}{|c|c|c|c|c|c|}
\hline Proyecto & Riesgos-PМBOK & Riesgos-CWW & Proyecto & Riesgos-РМВОК & Riesgos-CWW \\
\hline 1 & 6,50 & 3,63 & 8 & 10,00 & 5,02 \\
\hline 2 & 14,50 & 4,65 & 9 & 8,00 & 6,34 \\
\hline 3 & 10,50 & 1,69 & 10 & 14,00 & 5,10 \\
\hline 4 & 13,50 & 5,80 & 11 & 10,00 & 8,21 \\
\hline 5 & 8,00 & 3,63 & 12 & 7,00 & 5,46 \\
\hline 6 & 15,50 & 6,31 & 13 & 11,50 & 8,21 \\
\hline \multirow[t]{2}{*}{7} & 10,50 & 2,94 & 14 & 6,50 & 4,56 \\
\hline & & & Total & 146 & 71,55 \\
\hline
\end{tabular}

Tabla 4 - Valores de error cuadrático medio por proyectos

En el análisis de los resultados se identificó que el método Riesgos-PMBOK obtuvo un total de 146 en los errores cuadráticos, mientras que en el método Riesgos-CWW fue de 


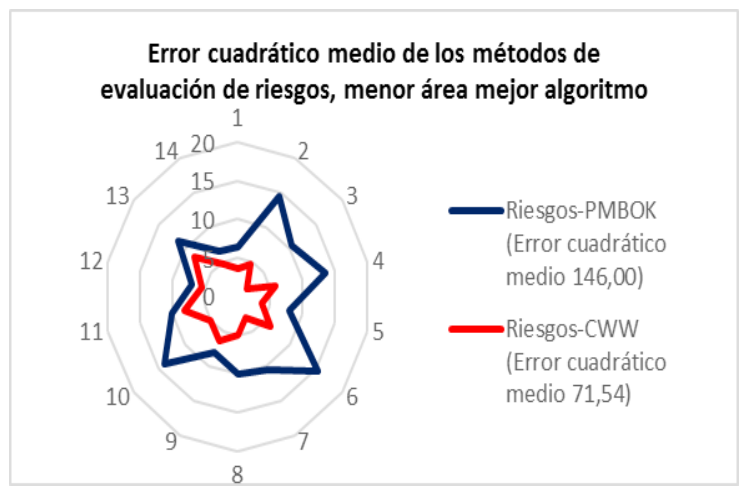

Figura 3 - Gráfico radial que representa el error cuadrático medio en la evaluación de riesgos

71.55. Como se muestra gráficamente en la Figura 3, los mejores resultados se obtienen con el método de evaluación Riesgos-CWW.

Como se puede ver entre las ventajas del método propuesto está la facilidad para interpretar los resultados al expresarse tanto la evaluación de los expertos como los resultados finales en palabras. El método propuesto además permite la valoración simultánea de múltiples expertos y como se puede ver los resultados de los dos métodos son diferentes. Ninguno de los riesgos evaluados con la evaluación tradicional PMBOK, tuvieron exposición alto a pesar de que la probabilidad o el impacto de algunos, era elevado. Además, el orden final luego de la evaluación, que es importante para la respuesta a los riesgos, fue también diferente, siendo superior el método propuesto.

Además, el método basado en computación con palabras resulta más intuitivo y permite una mejor interpretación de los resultados al usar términos lingüísticos en lugar de valores numéricos.

Con la aplicación de la propuesta en la herramienta GESPRO se han beneficiado un total de 14 centros de desarrollo de tecnologías de la información, en los cuales se gestionan más de 200 proyectos y donde convergen más de 500 usuarios de la herramienta. Además, se implementó el método en la compañía ecuatoriana QuitusServices, dedicada a la prestación de servicios de tecnologías de la información y las comunicaciones (QuitusServices, 2018). En dicha compañía, con la aplicación del método propuesto, se recuperó un monto de \$268 USD por concepto de ingresos por cliente según actividades en 1 mes.

\section{Conclusiones y trabajos futuros}

Los principales estándares de gestión de proyectos no tratan suficientemente el tema de aseguramiento de ingresos. Se identificó como tendencia en las estrategias de aseguramiento de ingresos la necesidad de combinar las estrategias reactivas, con estrategias activas y proactivas, con el objetivo de disminuir los tiempos de detección de las fugas de ingresos y prevenir las posibles fallas o acciones de fraudes. La propuesta combina técnicas de gestión de proyectos, análisis de riesgos y computación 
con palabras, para aplicar las técnicas de aseguramiento de ingresos bajo el enfoque proactivo. Respecto a los métodos de evaluación cualitativa de riesgos se concluye que el método basado en computación con palabras reporta mejores resultados que la técnica tradicional propuesta por el PMBOK. El método propuesto, se encuentra integrado a la plataforma GESPRO y se propone el uso de esta plataforma para su aplicación en la toma de decisiones por la versatilidad y las funcionalidades para el aseguramiento de ingresos.

En la instrumentación del método propuesto, sobre la toma de decisiones, pueden ser empleados sistemas de recomendaciones, entre otras técnicas de la computación emergente y se recomienda que se trabaje esta temática en investigaciones futuras. Se debe continuar investigando acerca de la aplicación del método propuesto en escenarios en tiempo real usando estrategias para el cómputo de altas prestaciones y potenciando la aplicación del método en un enfoque activo del aseguramiento de ingresos. Además, se recomienda realizar análisis comparativo con metodologías agiles como por ejemplo XP y SCRUM.

\section{Referencias}

Acosta, K. (2008). Aseguramiento de ingresos: una actividad fundamental en las empresas de telecomunicaciones. Ingeniería Industrial, 24(2), 1-6. Obtenido de http://www.redalyc.org/articulo.oa?id=360433566002

Castro, G. F. (2016). Platform for Project Evaluation Based on Soft-Computing Techniques. In: Communications in Computer and Information Science, CITI 2016. 658, págs. 226-240. Springer. doi:10.1007/978-3-319-48024-4_18

Castro, G. F. (2017). Aplicación de la minería de datos anómalos en organizaciones orientadas a proyectos. Revista Cubana de Ciencias Informáticas, 1o(Especial UCIENCIA), 195-209.

Delgado, R. (2011). La Dirección Integrada de Proyecto como Centro del Sistema de Control de Gestión en el Ministerio del Poder Popular para la Comunicación y la Información. Caracas, Venezuela: CENDA.

Duran D. (2017). Evaluación de mapas de competencias educativas: una propuesta difusa basada en 2-tuplas. Revista lbérica de Sistemas y Tecnologías de Información, (24), 22-38. doi:10.17013/risti.24.22-38

GRAPA. (2017). Professionalizing the Information, Communications and Technology Industry. The Global Revenue Assurance Professional Association (GRAPA), Obtenido de: http://www.grapatel.com

Herrera, F. M. (2000). A 2-tuple fuzzy linguistic representation model for computing with words. IEEE Transactions on Fuzzy Systems, 8(6), 746-752. doi:10.1109/91.890332

IPMA. (2016). International Project Management Association. Obtenido de http://www.ipma.world 
Johnsonbaugh, R. (1999). Matemática Discreta (4 ed.). México: Prentice Hall.

Khan, N. (2014). Internship Report on Revenue Assurance and Fraud Management ID: 10104009. BRAC Business School.

Leach, L. P. (2014). Critical chain project management (3rd edition). London: Artech House.

Mathkour H., A. G. (2008). A Risk Management Tool for Extreme Programming. IJCSNS International Journal of Computer Science and Network Security, 8(8), 326-333.

Mattison, R. (2005). The Telco Revenue Assurance Handbook. Oakwood Hills, Illinois, USA: XiT Press.

Merigó, J. Y. (2013). Norm aggregations and OWA operators. In Aggregation functions in theory and in practice. Advances in Intelligent Systems and Computing, 228, 141-151. doi:10.1007/978-3-642-39165-1_17

Phillips, J. (2013). Project Management Professional: Certification Study Guides (7 ed.). New York: McGraw-Hill Osborne Media.

PMI. (2017). A Guide to the Project Management Body of Knowledge. PMBOK ${ }^{\circledR}$ Guide (6 ed.). Pennsylvania, EE.UU.: Project Management Institute.

Prieto A., P. M. (2015). Propuesta de marco de mejora continua de gobierno TI en entidades financieras. Revista lbérica de Sistemas y Tecnologías de Información, (15), 51-67. doi:10.17013/risti.15.51-67

QuitusServices. (2018). Portal corporativo compañía de servicios informáticos. (Guayaquil, Ecuador) Obtenido de https://businessredmine.herokuapp.com/ portal/quitusservices

Schnoeller G., M. L. (2016). A strategy based on knowledge acquisition for management of requirements risks on distributed XP development. Revista lbérica de Sistemas y Tecnologías de Información, (20), 18-33. doi:10.17013/risti.20.18-33

Schwalbe, K. (2015). Information technology project management (7 ed.). Stamford: Cengage Learning.

SEI. (2010). CMMI para Desarrollo, Versión 1.3. Mejora de los procesos para el desarrollo de mejores productos y servicios. EE.UU.: Technical Report, Software Engineering Institute.

SGI. (2014). The CHAOS Manifesto. Obtenido de The Standish Group International: https://www.projectsmart.co.uk/white-papers/chaos-report.pdf

SGI. (2015). Standish Group 2015 Chaos Report. Obtenido de https://www.infoq.com/ articles/standish-chaos-2015

TMForum. (2015). Revenue Assurance practitioner blog: Do we need a new approach to revenue assurance in the digital world? \& Seeing is believing: Setting revenue assurance KPIs. Obtenido de https://inform.tmforum.org/ 
Verzuh, E. (2015). The fast forward $M B A$ in project management (5 ed.). New Jersey: Wiley.

Vondran, A. (2015). Metodologías ágiles de gestión de proyectos. Obtenido de https://www.linkedin.com/pulse/metodolog\%C3\%ADas-\%C3\%A1giles-degesti\% $3 \%$ B3n-proyectos-andre-vondran/

Zandhuis, A. S. (2012). ISO2150o: Guidance on Project Management: A Pocket Guide. Zaltbommel, The Netherlands: Van Haren Piblishing. 